\title{
Computed Tomography Enterography Findings Correlate with Tissue Inflammation, Not Fibrosis in Resected Small Bowel Crohn's Disease
}

\author{
Jeremy Adler, MD, MSc, ${ }^{*}$ Darashana R. Punglia, $M D_{,}^{+}$Jonathan R. Dillman, $M D_{,}^{\neq}$ \\ Alexandros D. Polydorides, MD, PhD, ${ }^{\S}$ Maneesh Dave, MBBS, MPH," Mahmoud M. Al-Hawary, MD," \\ Joel F. Platt, MD," Barbara J. McKenna, MD, and Ellen M. Zimmermann, MD**
}

\begin{abstract}
Background: It has become commonplace to categorize small intestinal Crohn's disease (CD) as "active" vs. "inactive" or "inflammatory" vs. "fibrotic" based on computed tomography enterography (CTE) findings. Data on histologic correlates of CTE findings are lacking. We aimed to compare CTE findings with histology from surgically resected specimens. We tested the hypothesis that CTE findings can distinguish tissue inflammation from fibrosis.
\end{abstract}

Methods: Patients who underwent CTE within 3 months before intestinal resection for CD were retrospectively studied. Radiologists blinded to history and histology scored findings on CTE. Pathologists blinded to history and imaging scored resected histology. We compared histology with CTE findings and radiologists assessment of whether the stricture was likely "active" or "inactive."

Results: In all, 22 patients met inclusion criteria. Inflammatory CTE findings correlated with histologic inflammation (rho $=0.52)$. Strictures believed to be "active" on CTE were more inflamed at histology $(P=0.0002)$. Strictures lacking inflammatory findings on CTE or considered "inactive" were not associated with greater histologic fibrosis or significant histologic inflammation. Upstream dilation was associated with greater tissue fibrosis in univariate $(P=0.014)$ but not in multivariate analysis $(P=0.53)$. Overall, histologic fibrosis correlated best with histologic inflammation $($ rho $=0.52$ ). Strictures on CTE with the most active disease activity also had the most fibrosis on histology.

Conclusions: CTE findings of mesenteric hypervascularity, mucosal hyperenhancement, and mesenteric fat stranding predict tissue inflammation. However, small bowel stricture without CTE findings of inflammation does not predict the presence of tissue fibrosis. Therefore, caution should be used when using CTE criteria to predict the presence of scar tissue.

(Inflamm Bowel Dis 2012;18:849-856)

Key Words: CT enterography, Crohn's disease, stricture, fibrosis, histology

Additional Supporting Information may be found in the online version of this article.

Received for publication April 21, 2011; Accepted May 20, 2011.

From the *Division of Pediatric Gastroenterology, Department of Pediatrics and Communicable Diseases, University of Michigan Health System, Ann Arbor, Michigan, ${ }^{\dagger}$ Department of Internal Medicine, University of Michigan Health System, Ann Arbor, Michigan, ${ }^{\dagger}$ Section of Pediatric Radiology, Department of Radiology, University of Michigan Health System, Ann Arbor, Michigan, ${ }^{\S}$ Department of Pathology, University of Michigan Health System, Ann Arbor, Michigan, "Department of Internal Medicine, Wayne State University, Detroit, Michigan, "Department of Radiology, Division of Abdominal Imaging, University of Michigan Health System, Ann Arbor, Michigan, ${ }^{* *}$ Division of Gastroenterology, Department of Internal Medicine, University of Michigan Health System, Ann Arbor, Michigan.

Funded by National Institutes of Health NIDDK R01 DK073992 (to E.M.Z.) and NICHD K12 HD028820 (to J.A.).

Reprints: Jeremy Adler, MD, MSc, University of Michigan, 1150 W. Medical Center Dr., A520B MSRB I, Box 5658, Ann Arbor MI 48109 (e-mail: jeradler@ umich.edu).

Copyright (C) 2011 Crohn's \& Colitis Foundation of America, Inc. DOI 10.1002/ibd.21801

Published online 24 June 2011 in Wiley Online Library (wileyonlinelibrary.com). rohn's disease $(\mathrm{CD})$ is a condition of chronic, trans$\checkmark$ mural bowel wall inflammation that can lead to luminal narrowing and stricture formation. The presence of an intestinal stricture can promote fistula and abscess formation proximal to the site of the stricture. ${ }^{1,2}$ Histologically, Crohn's strictures result from a combination of inflammation, smooth muscle hyperplasia, and fibrosis. In clinical practice, an intestinal stricture that is predominantly inflammatory often responds quickly to high-dose steroid therapy or therapy with potent antitumor necrosis factor alpha (TNF $\alpha)$ agents. If an intestinal stricture is primarily fibrotic, it is not likely to respond to antiinflammatory medical treatment. In patients with obstructive symptoms a trial of an antiinflammatory may relieve the intestinal obstruction, but exposes the patient to the numerous potential side effects of therapy, including deleterious effects of steroids on growth and development of children. $^{3-7}$ A stricture that does not respond to antiinflammatory therapy is presumed to be fibrotic and surgery is performed. The preoperative trial of medical therapy can delay 
TABLE 1. CTE Evaluation Scoring

\begin{tabular}{lcccc}
\hline Feature & 0 & 1 & 2 & 3 \\
\hline Abnormal mucosal/mural enhancement & None & Mild & Mod & Mod \\
Mesenteric hypervascularity & None & Mild & Mod & Marked \\
Mesenteric inflammatory stranding & None & Mild & Marked \\
Bowel wall thickening & None $(<3 \mathrm{~mm})$ & Mild $(3-5 \mathrm{~mm})$ & Mod $(5-7 \mathrm{~mm})$ & Marked $(>7 \mathrm{~mm})$ \\
\hline Mod, moderate. & & & & \\
\hline
\end{tabular}

surgical therapy and may increase postoperative complications. ${ }^{8,9}$ Numerous studies have demonstrated the ability of a variety of imaging modalities to predict disease activity ${ }^{10-14}$ and have attempted to distinguish between inflammatory and fibrotic strictures. ${ }^{15-19}$ However, to date no study has been able to accurately characterize the presence of or assess the degree of fibrosis in an intestinal stricture.

Computed tomography enterography (CTE) is a dedicated high-resolution CT examination with submillimeter collimation and low-Hounsfield unit oral contrast material that is optimized for small bowel evaluation. Intravenous and low Hounsfield unit oral contrast materials are thought to aid in distinguishing tissue types and, in particular, allow for assessment of fine details. Bowel wall inflammation is presumed to be present when radiologic findings of inflammation are present such as mucosal (or inner wall) hyperenhancement (including mural stratification), mesenteric fat stranding, and mesenteric hypervascularity (the so-called "comb sign"). ${ }^{20}$ Bowel wall fibrosis is presumed to be present when a segment of bowel wall appears abnormally thickened in the absence of radiologic signs of inflammation. CTE findings of mucosal hyperenhancement as well as mesenteric hypervascularity correlate with the Crohn's disease activity index (CDAI), ${ }^{13,21,22}$ and inflammation markers such as erythrocyte sedimentation rate (ESR) and C-reactive protein (CRP). ${ }^{23,24}$ The converse is often assumed to be true: luminal narrowing and wall thickening without mesenteric hypervascularity and mucosal enhancement in the setting of CD indicates a fibrotic stricture. No study has specifically tested the assumption that these CTE findings, presumed to represent fibrosis, actually correlate with histologic findings of fibrosis. ${ }^{25}$

We designed this study to retrospectively identify the specific radiologic correlates of intestinal strictures using histology as the reference standard. We tested the hypothesis that CTE findings can distinguish between bowel wall inflammation and fibrosis in small intestinal strictures in CD.

\section{PATIENTS AND METHODS}

\section{Patient Recruitment}

Patients who underwent CTE examinations were identified through the University of Michigan Health System
Department of Radiology adult and pediatric databases. Pertinent radiology reports and electronic medical records were reviewed for patients who underwent CTE between January 2003 and October 2007. All patients with CD who underwent surgical resection of a small bowel stricture for presumed CD within a 3-month period after CTE were eligible for inclusion in this study. Patients were excluded who were found to have another pathologic process in the resected tissue such as concomitant neoplasm or endometriosis. Patients were also excluded if tissue specimens were not available for review or if data were otherwise incomplete.

\section{CTE Review}

Two abdominal radiologists who were blinded to both the clinical history and to the pathologic findings retrospectively reviewed all CT scans. The findings that were scored included abnormal mucosal/mural enhancement, mesenteric hypervascularity, and mesenteric inflammatory fat stranding. Each of these CTE findings was graded on a Likert-like scale from 0 to 3 (Table 1). Bowel wall thickening was scored by categories of measured thickness (none $[<3 \mathrm{~mm}]$, mild [3-5 $\mathrm{mm}]$, moderate $[5-7 \mathrm{~mm}]$, or marked thickening [>7 mm]) (Fig. 1). Additional features were recorded including narrowest luminal diameter of the stricture and diameter of maximal upstream small bowel dilation.

A composite CTE inflammation score for each stricture was defined by the sum of scores for mucosal and mural hyperenhancement, mesenteric hypervascularity, and mesenteric inflammatory fat stranding. The radiologists additionally provided their global assessment of ileal inflammation (active disease vs. abnormal, but likely inactive). Disease was considered "active" if an involved abnormal bowel segment had mucosal hyperenhancement, mesenteric hypervascularity, and mesenteric fat stranding; and "inactive" if an involved abnormal bowel segment lacked these features.

\section{Histologic Evaluation}

Resected small bowel segments were retrieved and reviewed. Formalin-fixed, paraffin-embedded tissue sections from the point of maximal involvement or narrowing were stained with hematoxylin and eosin. Two gastrointestinal pathologists, blinded to the imaging findings, evaluated the resection specimens, scoring for degree of inflammation, degree of fibrosis, and the presence or absence of muscular 

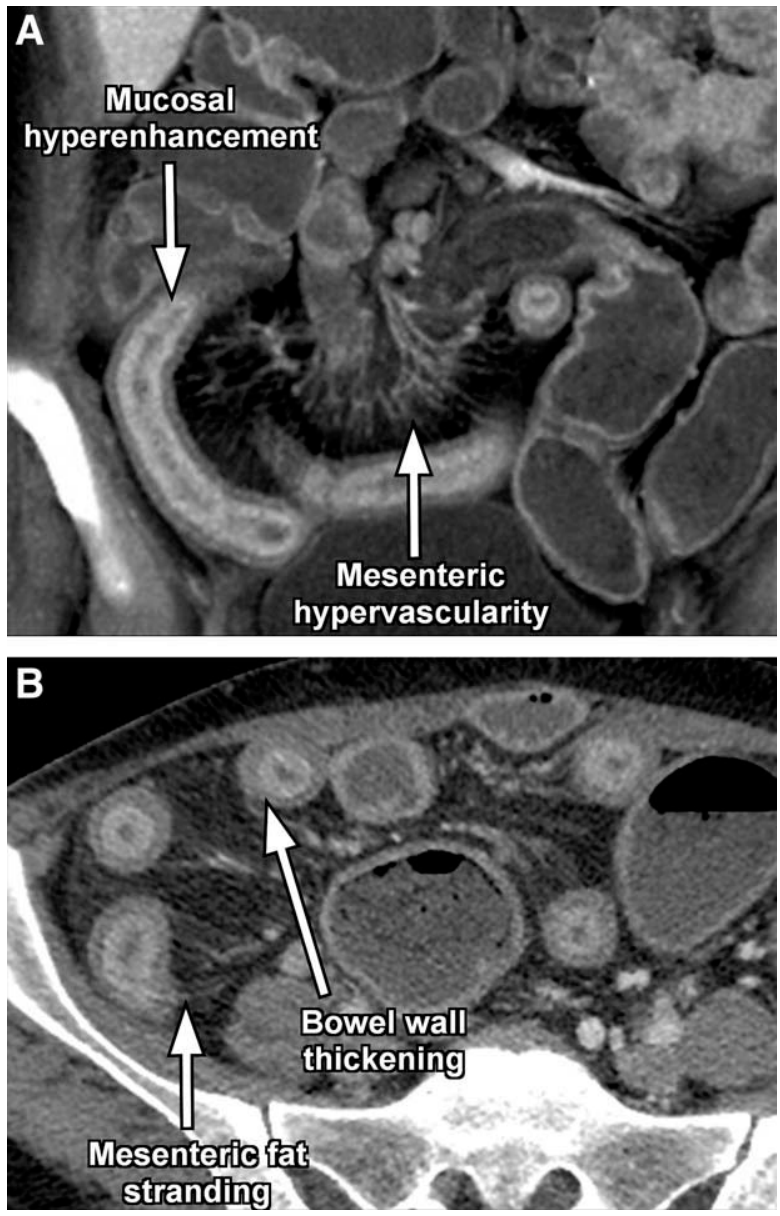

FIGURE 1. CTE findings. Illustrations of specific CTE findings. Bowel wall inflammation is seen as mucosal hyperenhancement, mesenteric hypervascularity (A), and mesenteric fat stranding (B). The finding of bowel wall thickening (B) and luminal narrowing in the absence of inflammatory findings has previously been felt to represent fibrosis.

hypertrophy (Table 2). The inflammation and fibrosis scores were adapted from histologic scores of small bowel CD previously published. ${ }^{26-28}$

\section{Stricture Identification}

In patients in whom multiple strictures were present, close review of the operative note was performed to determine if the stricture resected could be clearly identified on the CTE examination. In the two cases where the exact stricture that was resected could not be clearly determined, analysis was performed with and without inclusion of those patients (see Supporting Table 1).

\section{Statistical Evaluation}

Univariate comparisons were made with Spearman correlation when ordinal scales were involved. Continuous variables were compared with Pearson correlations. Associations between categorical variables were tested with a chi-square test. Bivariate comparisons were made with two-tailed Student's $t$-test. Multivariate ordered logistic regression (ologit) was performed for multivariate analysis where the dependent variable was an ordinal variable (such as tissue fibrosis score). Sex was represented as binary variable $(1=$ female, $0=$ male). Duration of disease was represented in years from diagnosis to CTE. Global assessment of stricture was represented as binary variable $(1=$ "active disease," $0=$ "abnormal, but likely inactive”). All statistical analyses were performed with Stata 11.1 for Mac (StataCorp, College Station, TX).

\section{Ethical Considerations}

This study was approved by the Institutional Review Board at the University of Michigan (IRB No. 2003-0306).

\section{RESULTS}

Of the 1387 patients who underwent CTE examinations from January 2003 to October 2007, 28 patients had $\mathrm{CD}$ and underwent surgery within 3 months after CTE (Fig. 2). Two patients were excluded due to either missing pathologic specimens or otherwise incomplete data. Of the remaining 26 patients, four were excluded based on the presence of neoplasm, lipoma, or endometriosis in their resected small bowel specimen. (The patient with lipoma had insufficient nonlipomatous tissue resected to be diagnostic.) The remaining 22 patients were included in the CTE-pathologic correlation portion of this study (Table 3). Of these 22 patients, 11 underwent resection for small bowel obstruction. Seven were described in the medical record as having medical refractory $\mathrm{CD}$ and four had surgery for fistulizing disease (although only three had mention of fistulas on the operative reports). Seven patients had more than one small bowel stricture present. In five of those patients the stricture that was resected

\section{TABLE 2. Histologic Evaluation Scoring}

\begin{tabular}{|c|c|}
\hline \multicolumn{2}{|c|}{ Fibrosis Grades } \\
\hline 0 & No fibrosis \\
\hline 1 & Minimal fibrosis in submucosa or subserosa \\
\hline 2 & $\begin{array}{l}\text { Increased submucosal fibrosis, septa into } \\
\text { muscularis propria }\end{array}$ \\
\hline 3 & $\begin{array}{l}\text { Septa through muscularis propria, increase } \\
\text { in subserosal collagen }\end{array}$ \\
\hline 4 & Significant transmural scar, marked subserosal collagen \\
\hline \multicolumn{2}{|c|}{ Inflammation Grades } \\
\hline 0 & No inflammation or distortion \\
\hline 1 & Lamina propria inflammation only \\
\hline 2 & Submucosal foci of inflammation \\
\hline 3 & Foci of transmural inflammation \\
\hline 4 & Significant, dissecting, confluent transmural inflammation \\
\hline \multicolumn{2}{|c|}{ Muscle } \\
\hline 0 & Normal thickness \\
\hline 1 & Increased thickness of muscularis propria layer \\
\hline
\end{tabular}




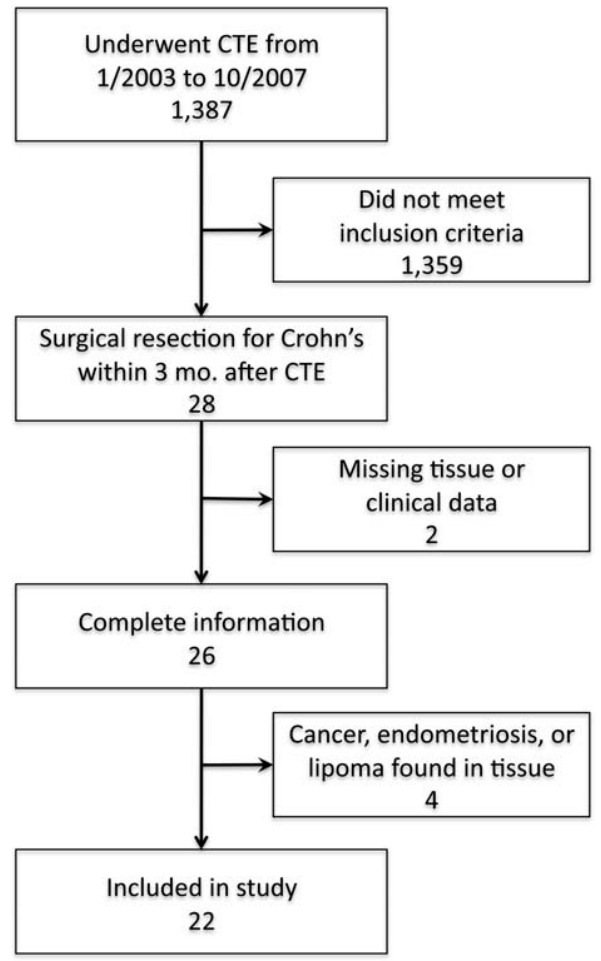

FIGURE 2. Consort diagram.

could be clearly identified in the operative report. In two patients the exact stricture resected could not be determined with certainty (Supporting Table 1).

The composite CTE radiologic inflammation score correlated well with the degree of histologic tissue inflammation $(n=22$; rho $=0.52 ; P=0.014$; Fig. 3). Individual CTE features that make up the components of the CTE inflammation score correlated as well, including mucosal hyperenhancement $($ rho $=0.89 ; P<0.0001)$, mesenteric hypervascularity (rho $=0.91 ; P<0.0001$ ), adjacent inflammatory fat stranding (rho $=0.82 ; P<0.0001)$, and wall thickening (rho $=0.83 ; P<0.0001$ ). The composite CTE radiologic inflammation score correlated with the degree of histologic tissue fibrosis $(n=22$; rho $=0.48$; $P=0.023$ ). These results did not change when the two patients with uncertain location of stricture resection were excluded from analysis (see Supporting Addendum).

The various CTE findings traditionally associated with bowel wall inflammation including mucosal hyperenhancement, bowel wall thickening, mesenteric hypervascularity, and adjacent mesenteric fat stranding each were found to increase with increasing degrees of inflammation in the corresponding resected tissue specimen (Fig. 4A). However, such a pattern was less apparent when comparing radiologic findings with degree of tissue fibrosis in resected specimens (Fig. 4B).

The presence of upstream dilation was associated with higher fibrotic content (histologic fibrosis score

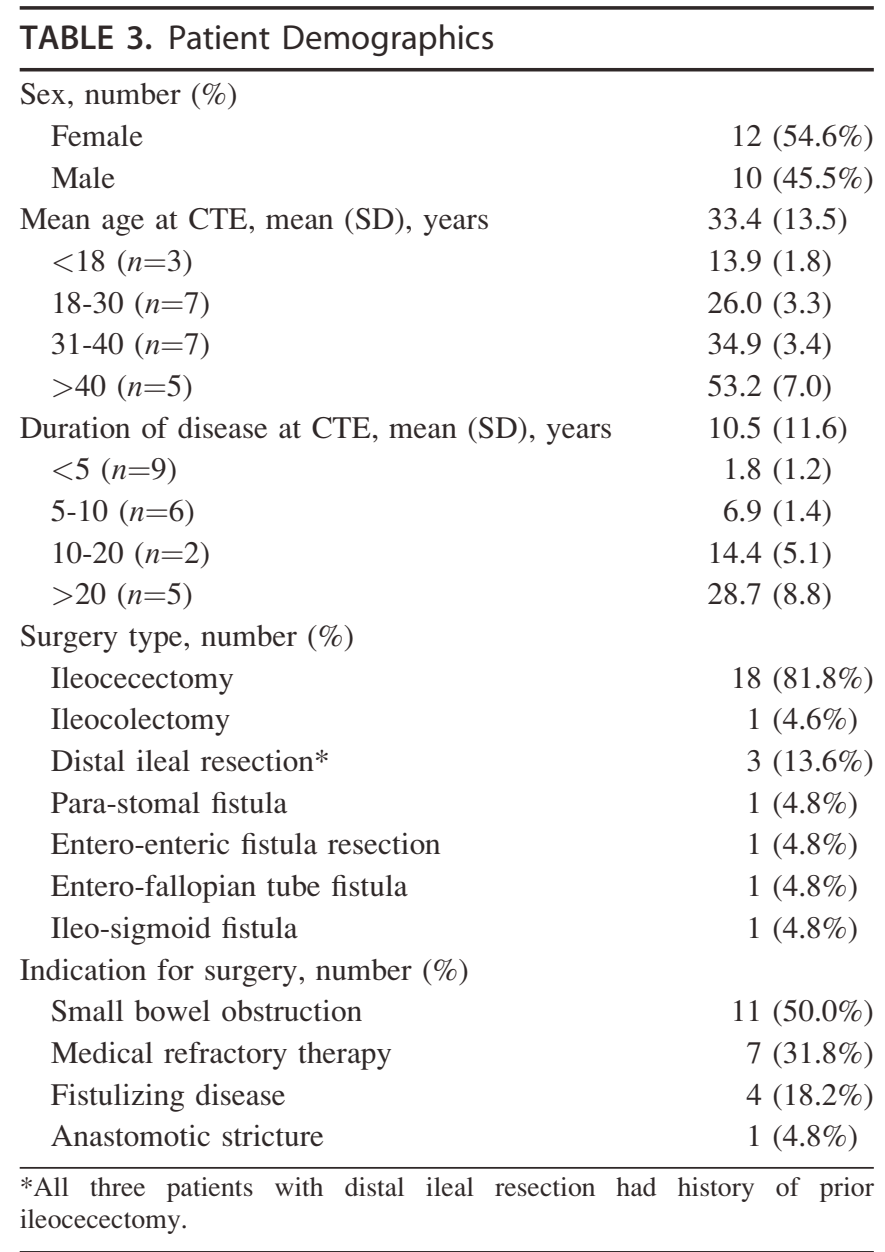

$3.69 \pm 0.17$ vs. $2.56 \pm 0.44 ; P=0.014)$ and with somewhat higher, although not statistically significant, inflammatory content (histologic inflammation score $3.23 \pm 0.20$ vs. $2.22 \pm 0.56 ; P=0.063)$ in resected specimens

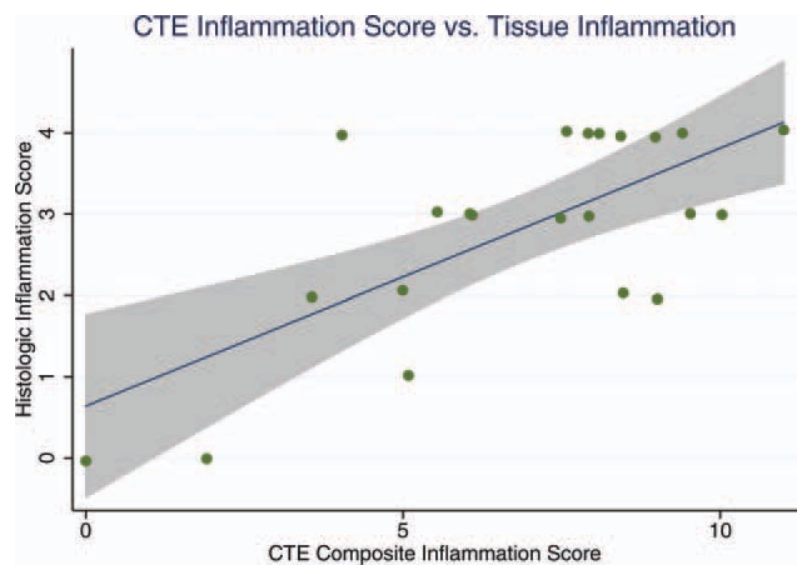

FIGURE 3. Composite CTE inflammation score correlates well with histologic score for tissue inflammation (rho $=0.52$; $P=0.014$ ). [Color figure can be viewed in the online issue, which is available at wileyonlinelibrary.com.] 

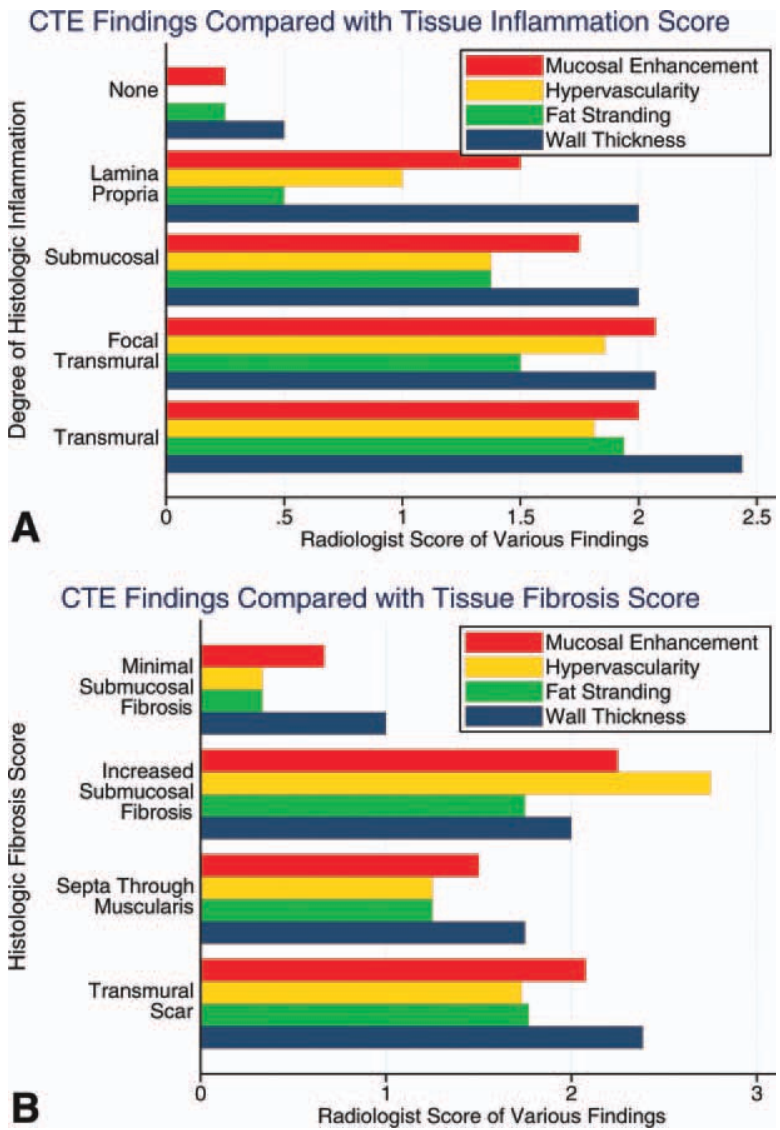

FIGURE 4. Radiologic findings associated with inflammation in stricture including mucosal hyperenhancement, mesenteric hypervascularity, inflammatory fat stranding, and bowel wall thickness are compared with degree of histologic tissue inflammation (A) and tissue fibrosis (B). Note that all radiologic scoring features increase as degree of tissue inflammation increases. However, radiologic scoring features do not follow a consistent pattern with increasing tissue fibrosis. [Color figure can be viewed in the online issue, which is available at wileyonlinelibrary.com.]

compared with those with the absence of appreciable upstream dilation (Fig. 5A). Neither the measured diameter of upstream dilation $\left(\chi^{2}=26.82 ; P=0.22\right)$ nor the tissue thickness $\left(\chi^{2}=22.43 ; P=0.097\right)$ of the stricture on CTE correlated with fibrotic content of the resected tissue.

When the radiologists global impression indicated "active disease" in the stricture, the corresponding tissue samples were found to have a greater degree of inflammation ( $n=17$; histologic inflammation score $3.29 \pm 0.19$ ) than those strictures felt to be "abnormal, but likely inactive disease" $(n=5$; histologic inflammation score $1.20 \pm$ $0.58 ; P=0.0002$ ). Those strictures felt to represent "active disease" also had a higher degree of tissue fibrosis than those felt to be "abnormal, but likely inactive" $(n=22$; $3.65 \pm 0.17$ vs. $1.80 \pm 0.49 ; P=0.0002$ ) (Fig. 5B). Those strictures felt to represent "inactive disease" were associated with the least tissue fibrosis.
When histologic tissue inflammation and fibrosis were compared with one another, all specimens that were more fibrotic were also found to be more inflamed. Those that were less fibrotic were also less inflamed. There were no fibrotic specimens that were not inflamed, and no inflamed specimens that were not fibrotic (Table 4). The degree of tissue inflammation correlates best with the degree of tissue fibrosis $(n=22$; rho $=0.52 ; P=0.014$; Fig. 6$)$.

Ordered logistic regression was performed with radiologic findings (mucosa/mural enhancement, mesenteric hypervascularity, mesenteric inflammatory stranding, bowel wall thickening, upstream dilation [presence or absence], duration of disease, and sex) as independent variables, and histologic tissue fibrosis score as the ordinal dependent
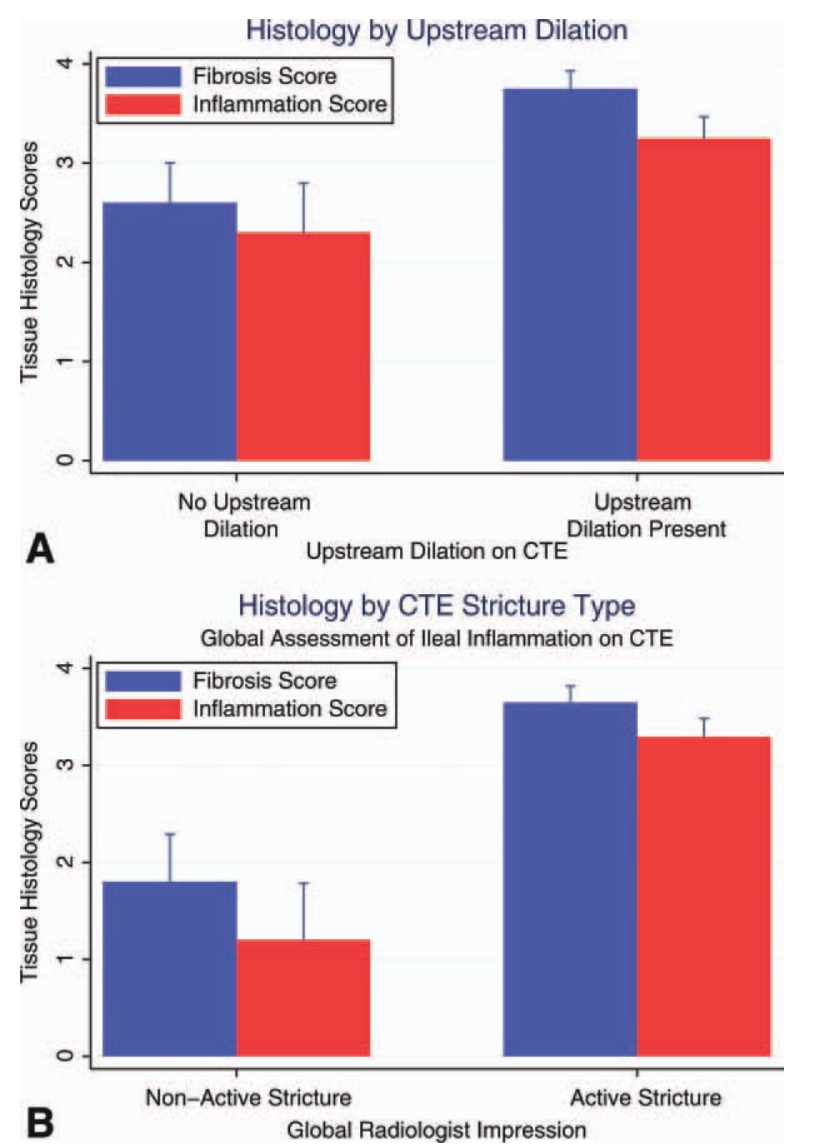

FIGURE 5. Resected stricture tissue histologic findings were compared in patients with and without upstream dilation on CTE (A) and between patients with and without active disease identified in the stricture on CTE (B). Patients with upstream dilation had greater tissue fibrosis ( $3.69 \pm 0.17$ vs. $2.56 \pm 0.44 ; P=0.014)$ and inflammation ( $3.23 \pm 0.20$ vs. $2.22 \pm 0.56 ; P=0.063)$ in resected specimens than those without upstream dilation. Patients in whom the radiologists global impression was that the stricture was 'active' the highest degree of tissue fibrosis $(3.65 \pm 0.17$ vs. $1.80 \pm 0.49$; $P=0.0002)$ and inflammation (3.29 \pm 0.19 vs. $1.20 \pm 0.58 ; P=$ 0.0002) compared with strictures described as 'not active.' [Color figure can be viewed in the online issue, which is available at wileyonlinelibrary.com.] 
TABLE 4. Tissue Fibrosis by CTE Findings

\begin{tabular}{|c|c|c|c|c|c|c|}
\hline \multirow[b]{2}{*}{ Histologic Fibrosis Score } & \multicolumn{5}{|c|}{ Histologic Inflammation Score } & \multirow[b]{2}{*}{ Total } \\
\hline & None & $\begin{array}{l}\text { Lamina Propria } \\
\text { Inflammation }\end{array}$ & $\begin{array}{l}\text { Submucosal } \\
\text { Inflammation }\end{array}$ & $\begin{array}{l}\text { Focal Transmural } \\
\text { Inflammation }\end{array}$ & $\begin{array}{l}\text { Transmural } \\
\text { Inflammation }\end{array}$ & \\
\hline Minimal submucoslal fibrosis & 2 & 1 & 0 & 0 & 0 & 3 \\
\hline Increased submucosal fibrosis & 0 & 0 & 0 & 1 & 1 & 2 \\
\hline Septa through muscularis & 0 & 0 & 1 & $3 *$ & 0 & 4 \\
\hline Transmural scar & 0 & 0 & $3 *$ & 3 & 7 & 13 \\
\hline Total & 2 & 1 & 4 & 7 & 8 & 22 \\
\hline
\end{tabular}

variable. The results with and without considering interaction terms are represented in Table 5. Hyperenhancement is positively associated with a tendency toward greater tissue fibrosis. For a one unit increase in degree of postcontrast enhancement, the expected ordered odds increases by 8.52 as you move to the next higher category of fibrosis (for example, from minimal submucosal fibrosis to increased submucosal fibrosis). Mesenteric hypervascularity is negatively associated with a tendency toward greater tissue fibrosis. For a one unit increase in mesenteric hypervascularity, the expected ordered log odds decreases by 9.96 as you move to the next higher category of tissue fibrosis. Mesenteric inflammatory fat stranding is positively associated with tissue fibrosis, with a one unit increase being associated with a tendency toward greater tissue fibrosis category (ordered log odds 5.93). There was no statistically significant effect of bowel wall thickening, upstream dilation, duration of disease, or sex on tissue fibrosis. The adjusted pseudo $\mathrm{R}^{2}$ of $0.5740(P=$ 0.0002 ) indicates that $57.4 \%$ of the variability in tissue fibrosis score is explained by this model. Predicted probabilities for transmural fibrotic scar found in the resected specimen based on mesenteric hypervascularity are represented in Supporting Figure 1 (also see Supporting Table 2). All analyses were repeated with the exclusion of the two patients with whom ambiguity existed regarding precisely which stricture was resected. The results of these analyses were not significantly different to the above findings (Supporting Table 3).

\section{DISCUSSION}

CTE provides exquisitely detailed images of the small bowel. Numerous studies have demonstrated the ability of CTE to detect small bowel disease activity in patients with $\mathrm{CD}$, and CTE findings have been shown to correlate with serologic markers of inflammation. ${ }^{23,24}$ CTE findings also correlate with endoscopic disease activity. ${ }^{23,29-31}$

However, the general assumptions that are commonly made regarding the tissue composition of small intestinal strictures due to $\mathrm{CD}$ based on CTE findings have not been validated. ${ }^{25}$ In this study we demonstrated that the CTE find- ings of bowel wall inflammation do, in fact, indicate the presence of tissue inflammation. However, we did not find a strong correlation between the radiologic findings presumed to be suggestive of fibrosis and the presence of histologic fibrosis. In fact, the only feature on CTE that predicted the presence of small bowel fibrosis in multivariate analysis was mesenteric hypervascularity. Mesenteric hypervascularity itself correlates better with the presence of tissue inflammation than with tissue fibrosis. This appears to be due to, at least in part, the pathologic relationship between inflammation and fibrosis. Histologic inflammation and fibrosis are strongly correlated with one another, and do not appear to be separable entities. Our findings agree with the findings of Zappa et al, ${ }^{32}$ who previously raised the issue that inflammation and fibrosis in $\mathrm{CD}$ are inseparably linked. It is in fact likely that CTE cannot assess the presence of fibrosis with or without associated inflammation because fibrosis and inflammation do not exist as mutually exclusive stricture types.

This study was limited by a relatively small sample size. However, the range of abnormal features in the CTE examinations in this series is typical of the spectrum of

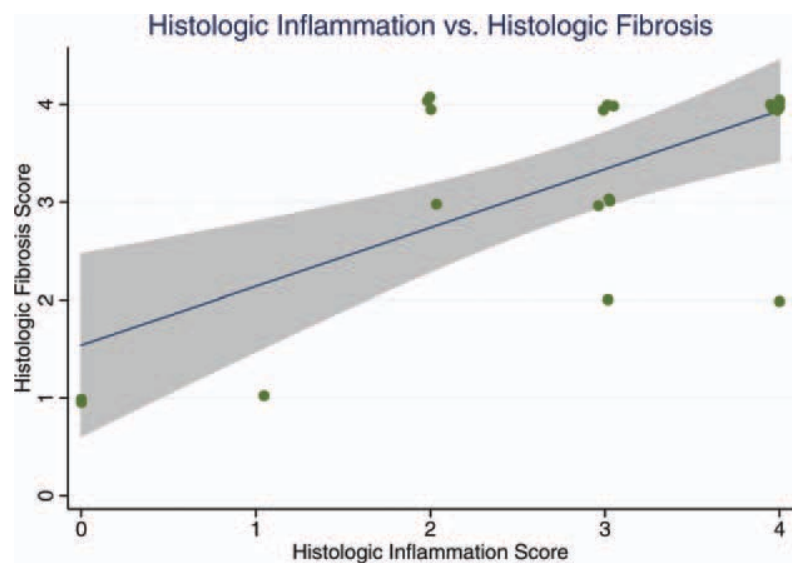

FIGURE 6. Histologic score for inflammation and fibrosis correlate with one another in resected tissue samples (rho $=0.52 ; P=$ 0.014). Note data points were subject to jitter(7) in Stata for ease of visualization. [Color figure can be viewed in the online issue, which is available at wileyonlinelibrary.com.] 
TABLE 5. Ordered Logistic Regression for Tissue Fibrosis

\begin{tabular}{lrcrrr}
\hline Parameter & Coefficient & Standard Error & 95\% CI for Parameter & Test Statistic & $P$-value \\
\hline Enhancement & 8.52 & 4.24 & 0.21 & 16.83 & 2.01 \\
Hypervascularity & -9.96 & 4.08 & -17.95 & -1.97 & -2.44 \\
Stranding & 5.93 & 2.89 & 0.27 & 11.58 & 2.05 \\
Thickening & 3.47 & 1.88 & -0.21 & 7.16 & 0.044 \\
Upstream dilation & 0.96 & 1.53 & -2.03 & 3.95 & 0.040 \\
Disease duration & -0.15 & 0.10 & -0.34 & 0.048 & 0.063 \\
Sex & 0.80 & 1.65 & -2.43 & 4.03 & -1.48 \\
\hline
\end{tabular}

SEM, standard error of the mean; CI, confidence interval.

abnormalities seen in our clinical practice. ${ }^{21}$ Our sample also lacked purely fibrotic strictures and the most fibrotic strictures also had significant inflammation. It is possible that $\mathrm{CD}$ patients with fibrotic strictures with no inflammation exist, but our experience and that of others would suggest that this is likely very rare. ${ }^{32}$ Ideally, CTE would have been performed on the same day or the day prior to surgical resection, but for practical reasons we chose to study patients with CTE within 3 months of resection. The length of time from CTE to surgery allows for the possibility of intervening worsening of disease or change in the histologic tissue composition. However, bowel wall fibrosis, if reversible, would not be expected to regress significantly in this time frame, and if anything might progress. Therefore, our results may underrepresent the degree of fibrosis present at the later time of surgery, not overrepresent the differences. Finally, it is possible that CTE could be better able to distinguish between inflammation and fibrosis in less fibrotic bowel. However, patients with lesser degrees of fibrosis would not be expected to undergo surgical resection, and therefore would not be included as part of this study. Our study design is not able to address this question.

Despite these limitations, this is the largest study to date investigating the relationship between findings on CTE of patients with $\mathrm{CD}$ and the corresponding histologic findings in resected specimens from the same patients. Our data support that the CTE findings of mucosal hyperenhancement, mesenteric hypervascularity, and adjacent inflammatory fat stranding correlate with the degree of tissue inflammation in resected specimens. However, bowel wall thickening without inflammatory signs does not appear to be a useful predictor of tissue fibrosis.

\section{REFERENCES}

1. Zimmermann EM, Lund PK. Fibrogenesis in inflammatory bowel disease. In: Kirsner J, ed. Inflammatory Bowel Disease. Philadelphia: W.B. Saunders; 2003.

2. Pucilowska JB, Williams KL, Lund PK. Fibrogenesis. IV. Fibrosis and inflammatory bowel disease: cellular mediators and animal models. Am J Physiol. 2000;279:G653-659.
3. Kumagai S, Kawano S, Atsumi T, et al. Vertebral fracture and bone mineral density in women receiving high dose glucocorticoids for treatment of autoimmune diseases. $J$ Rheumatol. 2005;32:863-869.

4. Bollet AJ, Black R, Bunim JJ. Major undesirable side-effects resulting from prednisolone and prednisone. JAMA. 1955;158:459-463.

5. Whitworth JA. Adrenocorticotrophin and steroid-induced hypertension in humans. Kidney Int. 1992;37:S34-37.

6. Saag KG, Koehnke R, Caldwell JR, et al. Low dose long-term corticosteroid therapy in rheumatoid arthritis: an analysis of serious adverse events. Am J Med. 1994;96:115-123.

7. Brenner DJ, Hall EJ. Computed tomography—an increasing source of radiation exposure. $N$ Engl J Med. 2007;357:2277-2284.

8. Yamamoto T, Allan RN, Keighley MR. Risk factors for intra-abdominal sepsis after surgery in Crohn's disease. Dis Colon Rectum. 2000; 43:1141-1145.

9. Alves A, Panis Y, Bouhnik Y, et al. Risk factors for intra-abdominal septic complications after a first ileocecal resection for Crohn's disease: a multivariate analysis in 161 consecutive patients. Dis Colon Rectum. 2007;50:331-336.

10. Gervais DA, Hahn PF, O’Neill MJ, et al. Percutaneous abscess drainage in Crohn disease: technical success and short- and long-term outcomes during 14 years. Radiology. 2002;222:645-651.

11. Post S, Betzler M, von Ditfurth B, et al. Risks of intestinal anastomoses in Crohn's disease. Ann Surg. 1991;213:37-42.

12. Steffes C, Fromm D. Is preoperative parenteral nutrition necessary for patients with predominantly ileal Crohn's disease? Arch Surg. 1992; 127:1210-1212.

13. Bodily KD, Fletcher JG, Solem CA, et al. Crohn disease: mural attenuation and thickness at contrast-enhanced CT enterography-correlation with endoscopic and histologic findings of inflammation. Radiology. 2006;238:505-516.

14. Taylor SA, Punwani S, Rodriguez-Justo M, et al. Mural Crohn disease: correlation of dynamic contrast-enhanced MR imaging findings with angiogenesis and inflammation at histologic examination-pilot study. Radiology. 2009;251:369-379.

15. Aro P, Ronkainen J, Talley NJ, et al. Body mass index and chronic unexplained gastrointestinal symptoms: an adult endoscopic population based study. Gut. 2005;54:1377-1383.

16. Joo JS, Agachan F, Wexner SD. Laparoscopic surgery for lower gastrointestinal fistulas. Surg Endosc. 1997;11:116-118.

17. Young S, Smith IS, O'Connor J, et al. Results of surgery for Crohn's disease in the Glasgow region, 1961-70. Br J Surg. 1975;62:528-534.

18. Lazarev M, Ullman T, Schraut WH, et al. Small bowel resection rates in Crohn's disease and the indication for surgery over time: experience from a large tertiary care center. Inflamm Bowel Dis. 2010;16:830-835.

19. Soyer P, Boudiaf M, Sirol M, et al. Suspected anastomotic recurrence of Crohn disease after ileocolic resection: evaluation with CT enteroclysis. Radiology. 2010;254:755-764.

20. Liu YB, Liang $\mathrm{CH}$, Zhang ZL, et al. Crohn disease of small bowel: multidetector row CT with CT enteroclysis, dynamic contrast enhancement, CT angiography, and 3D imaging. Abdom Imaging. 2006;31: $668-674$. 
21. Leighton JA, Shen B, Baron TH, et al. ASGE guideline: endoscopy in the diagnosis and treatment of inflammatory bowel disease. Gastrointest Endosc. 2006;63:558-565.

22. von Ahsen N, Armstrong VW, Behrens C, et al. Association of inosine triphosphatase $94 \mathrm{C}>\mathrm{A}$ and thiopurine S-methyltransferase deficiency with adverse events and study drop-outs under azathioprine therapy in a prospective Crohn disease study. Clin Chem. 2005;51:2282-2288.

23. Higgins LM, McDonald SA, Whittle N, et al. Regulation of $\mathrm{T}$ cell activation in vitro and in vivo by targeting the OX40-OX40 ligand interaction: amelioration of ongoing inflammatory bowel disease with an $\mathrm{OX} 40-\mathrm{IgG}$ fusion protein, but not with an OX40 ligand-IgG fusion protein. J Immunol. 1999;162:486-493.

24. Solem CA, Loftus EV Jr, Tremaine WJ, et al. Correlation of C-reactive protein with clinical, endoscopic, histologic, and radiographic activity in inflammatory bowel disease. Inflamm Bowel Dis. 2005;11: 707-712.

25. Lahat A, Chowers Y. The patient with recurrent (sub) obstruction due to Crohn's disease. Best Pract Res. 2007;21:427-444.

26. Maconi G, Carsana L, Fociani P, et al. Small bowel stenosis in Crohn's disease: clinical, biochemical and ultrasonographic evalu- ation of histological features. Aliment Pharmacol Ther. 2003;18: 749-756.

27. Theiss AL, Fuller CR, Simmons JG, et al. Growth hormone reduces the severity of fibrosis associated with chronic intestinal inflammation. Gastroenterology. 2005;129:204-219.

28. Chiorean MV, Sandrasegaran K, Saxena R, et al. Correlation of CT enteroclysis with surgical pathology in Crohn's disease. Am J Gastroenterol. 2007; 102:2541-2550.

29. Ebinger M, Leidl R, Thomas S, et al. Cost of outpatient care in patients with inflammatory bowel disease in a German University Hospital. J Gastroenterol Hepatol. 2004;19:192-199.

30. Konig HH, Ulshofer A, Gregor M, et al. Validation of the EuroQol questionnaire in patients with inflammatory bowel disease. Eur J Gastroenterol Hepatol. 2002;14:1205-1215.

31. Schmid RM, Adler G, Liptay S. Activation of NFkappaB in inflammatory bowel disease. Gut. 1998;43:587-588.

32. Zappa M, Stefanescu C, Cazals-Hatem D, et al. Which magnetic resonance imaging findings accurately evaluate inflammation in small bowel Crohn's disease? A retrospective comparison with surgical pathologic analysis. Inflamm Bowel Dis. 2011;17:984-993. 